\title{
The consequences of modeling autocorrelation when synthesizing single-case studies using a three-level model
}

\author{
Merlande Petit-Bois $^{1,4}$ • Eun Kyeng Baek ${ }^{1}$ - Wim Van den Noortgate ${ }^{2}$. \\ S. Natasha Beretvas ${ }^{3}$. John M. Ferron ${ }^{1}$
}

Published online: 13 October 2015

(C) Psychonomic Society, Inc. 2015

\begin{abstract}
Results from single-case studies are being synthesized using three-level models in which repeated observations are nested in participants, which in turn are nested in studies. We examined the performance of these models under conditions in which the errors associated with the repeated observations (the Level-1 errors) were assumed to be first-order autoregressive. Monte Carlo methods were used to examine conditions in which the first-order autoregressive assumption was accurate, conditions in which it represented an overspecification because the errors were actually independent, and conditions in which it represented a misspecification because the errors were generated on the basis of a moving-average model. Conditions also varied the series lengths, the numbers of participants per study, the numbers of studies per meta-analysis, the variances between the participants within studies, and the variances between studies. Fixed effects (e.g., the average treatment effect for the intervention and the average treatment effect for the trend) tended to be unbiased, and confidence intervals for the fixed effects tended to be accurate even when the error covariance model was overspecified or misspecified. The variance components, particularly at Levels 2 and 3, showed substantial bias.
\end{abstract}

Merlande Petit-Bois

mpetitbo@usf.edu

University of South Florida, Tampa, FL, USA

Katholieke Universiteit Leuven, Leuven, Belgium

University of Texas, Austin, TX, USA

4 Department of Educational Measurement and Research, University of South Florida, Tampa, FL, USA
Keywords Meta-analysis · Single-case designs $\cdot$ Multilevel modeling $\cdot$ Autocorrelation

Meta-analytic procedures allow researchers to quantitatively synthesize past research results and provide evidence for best practices (Hedges \& Olkin, 1985). Meta-analyzing single-case research has become increasingly popular. Several methods have been proposed to aggregate data across single-case studies. Beretvas and Chung (2008) conducted a narrative review of 25 single-case meta-analyses and found that most of the single-case or single-participant meta-analysts were using nonparametric approaches. They further concluded that metaanalysts were using the simplest indicators as effect size measures and nonparametric methods, such as the percentage of nonoverlapping data (PND; Scruggs, Mastropieri, \& Castro, 1987 ) and the percentage of all nonoverlapping data (PAND; Parker, Hagan-Burke, \& Vannest, 2007). Although these metrics are appealing, they are limited in that they may not have formally developed standard errors, which makes it difficult to make inferential statements about the treatment effects.

Another method that has been suggested to synthesize data across single-case studies is multilevel modeling (Van den Noortgate \& Onghena, 2008). A multilevel model can be applied to the raw data in meta-analyses in which the outcomes are measured on the same scale across single-case studies (for an example application, see Moeyaert, Ferron, Beretvas, \& Van den Noortgate, 2014), or a multilevel model can be applied after the data have been standardized (Moeyaert, Ugille, Ferron, Beretvas, \& Van den Noortgate, 2013a). The use of multilevel modeling provides an estimate of the average treatment effect, and statistical inferences can be made about that effect. Additionally, multilevel models are flexible enough to handle various complexities that may arise in the modeling of single-case data. Of particular interest in this study is that the 
use of multilevel modeling allows researchers to specify various error structures and to estimate treatment effects and their variances under conditions in which the meta-analyst assumes the errors of the repeated observations are serially dependent (as opposed to independent).

\section{Issue of autocorrelation}

In single-case data, it is plausible that the errors that are closer in time would be more similar, because nonmodeled factors (e.g., sickness or family loss) affecting the scores might be more similar at closer points in time. This autocorrelation in the errors means that the assumption of independence, which may be plausible in a between-subjects design, is questionable for single-subject designs. Kratochwill et al. (1974) provided a classic example of observed autocorrelation in behavioral data, and more recently Shadish and Sullivan (2011) conducted a survey of single-case studies in which they found that the meta-analytic mean of 800 autocorrelation estimates to be .20 , after adjusting the estimates for bias.

Simply assuming that the within-person errors are uncorrelated, when in fact they are not, has been shown to lead to inflated Type I error rates for tests of treatment effects in single-level regression models applied to the interrupted time-series data from a single case (Greenwood \& Matyas, 1990; Toothaker, Banz, Noble, Camp, \& Davis, 1983). If the autocorrelation is positive, standard errors tend to be smaller, and the results of the $t$ or $F$ tests will be inflated or biased in a positive manner. This inflation increases the Type I error rate (i.e., too often showing an effect when there actually is none). Conversely, if the correlation between the errors is negative, then the standard errors will be too large, causing the $t$ or $F$ test statistics to be smaller. This in turn, will increase Type II error rates, or too often failing to show an effect when there actually is one (Kazdin, 2011).

Ignoring positive autocorrelation in two-level models for multiple-participant single-case designs (e.g., the multiple-baseline design) has been shown to bias the variance components and to reduce confidence interval coverage for estimates of the average treatment effect (Ferron, Bell, Hess, Rendina-Gobioff, \& Hibbard, 2009). These results are consistent with general methodological work on the application of multilevel models to longitudinal data, which has found that misspecifying the Level1 error structure tends to bias the variance components and can impact fixed-effect inferences (Ferron,Dailey, \& Yi, 2002; Kwok, West, \& Green, 2007). Given that singlecase data may be autocorrelated and the negative consequences of ignoring autocorrelation, it is often recommended that single-case researchers choose an analysis method appropriate for autocorrelated data (Kazdin, 2011; Kratochwill et al., 2010).
One approach to the issue of autocorrelation is to select an analysis method that does not require assumptions about autocorrelation. Randomization tests (Bulté \& Onghena, 2008, 2009; Heyvaert \& Onghena, 2014) can be used to test for treatment effects without making autocorrelation assumptions, and have been shown to control the Type I error rate regardless of the level of autocorrelation (Ferron \& Ware, 1995; Ferron \& Onghena, 1996; Levin, Ferron, \& Kratochwill, 2012). Furthermore, randomization tests can be used with a variety of test statistics (Heyvaert \& Onghena, 2014) and graphical displays (Ferron \& Jones, 2006), and the $p$ values from randomization tests can be combined across studies in research syntheses (Edgington \& Onghena, 2007). Alternatively, overall effect sizes can be estimated using nonoverlap statistics that do not make assumptions about autocorrelation, such as the PND, PAND, nonoverlap of all pairs (Parker, Vannest, \& Davis, 2011a), and TauU, which can be adjusted for baseline trend (Parker, Vannest, Davis, \& Sauber, 2011b). These indices can then be combined across cases and studies in research syntheses.

A second approach to the autocorrelation issue is to explicitly account for autocorrelation in a statistical model. Approaches that explicitly account for autocorrelation include the single-case $d$-statistic (Hedges, Pustejovsky, \& Shadish, 2012, 2013), generalized least squares regression (Swaminathan, Rogers, \& Horner, 2014), simulation modeling (Borckardt \& Nash, 2014), and the inclusion of an autocorrelation parameter in the estimation of multilevel models (Ferron et al., 2009; Van den Noortgate \& Onghena, 2003). This study focuses in on the use of multilevel modeling for the meta-analysis of single-case data, the synthesis of data from multiple cases and from multiple studies.

\section{Three-level meta-analytic model for single-case designs}

Van den Noortgate and Onghena (2008) proposed that if raw data from several single-case designs are used in a meta-analysis, scores can vary at each of the three levels: over occasions (Level 1), across participants from the same study (Level 2), and across studies (Level 3). Equations 1-3 show the model specification for a simple three-level model.

Level-1 Equation:

$$
\begin{aligned}
\mathrm{Y}_{i j k}= & \beta_{0 j k}+\beta_{1 j k}{ }^{*} \text { phase }_{i j k}+\beta_{2 j k}{ }^{*} \text { time }_{i j k} \\
& +\beta_{3 j k}{ }^{*} \text { phase }_{i j k}{ }^{*} \text { time }_{i j k}^{\prime}+e_{i j k}, e_{i j k} \sim N\left(0, \Sigma_{e}\right)
\end{aligned}
$$

Level-2 Equations:

$$
\begin{aligned}
& \beta_{0 j k}=\theta_{00 k}+u_{0 j k} \\
& \beta_{1 j k}=\theta_{10 k}+u_{1 j k} \\
& \beta_{2 j k}=\theta_{20 k}+u_{2 j k} \\
& \beta_{3 j k}=\theta_{30 k}+u_{3 j k}
\end{aligned}
$$


Level-3 Equations:

$$
\begin{aligned}
\theta_{00 k} & =\boldsymbol{r}_{000}+v_{00 k} \\
\theta_{10 k} & =\boldsymbol{r}_{100}+v_{10} \\
\theta_{20 k} & =\boldsymbol{r}_{200}+v_{20 k} \\
\theta_{30 k} & =\boldsymbol{r}_{300}+v_{30 k}
\end{aligned}
$$$$
\left[\begin{array}{c}
v_{0 j k} \\
v_{1 j k} \\
v_{2 j k} \\
v_{3 j k}
\end{array}\right] \sim N\left(0, \Sigma_{v}\right)
$$

The score for the dependent variable on measurement occasion $i$ for subject $j$ of study $k$ is $Y_{i j k}$. The phase variable is a dummy-coded indicator reflecting whether the observation is in the baseline phase phase $_{i j k}=0$ ) or the intervention phase (phase $_{i j k}=1$ ). The time variable can be centered in a variety of ways that may be helpful for interpreting the model parameters (Baek et al., 2014). Specifically, for this study, the time variable is uncentered for the baseline phase's slope, such that on the first measurement occasion in the baseline phase, time $_{i j k}$ $=0$, and thus $\beta_{0 j k}$ is the expected value at the beginning of the study. However, the time' variable in the interaction term, phase $e_{i j k}{ }^{*} t i m e^{\prime}{ }_{i j k}$ is coded such that time ${ }_{i j k}^{\prime}=0$ for the first observation in the intervention phase. Therefore, $\beta_{1 j k}$ is the difference between the expected score during the baseline phase if it were extended one observation into treatment and the expected score of the treatment phase at this same point in time. As a result, $\beta_{1 j k}$ refers to the immediate level change associated with the intervention (Huitema \& McKean, 2000). $\beta_{2 j k}$ represents the linear trend during the baseline phase, and the linear trend for the treatment phase will be $\beta_{3 j k}$ higher. In other words, $\beta_{3 j k}$ indicates the effect of the intervention on the trend or the shift in slopes. Both $\beta_{1 j k}$ and $\beta_{3 j k}$ are needed to fully describe the treatment effect.

The error in the Level-1 model $\left(e_{i j k}\right)$ is assumed to be normally distributed with some covariance $\Sigma$. A variety of alternative structures for $\Sigma$ can be assumed (Wolfinger, 1993), including an independent structure $\left(\sigma^{2} \mathrm{I}\right)$ and a first-order autoregressive structure $[\mathrm{AR}(1)]$. Figure 1 shows the covariance structure of three specifications for the Level-1 error structure. The first specification, $\Sigma=\sigma^{2}$ I, represents a completely independent structure, with none of the errors being correlated. The next example, the first-order autoregressive structure, $\Sigma=\operatorname{AR}(1)$, illustrates that the errors are correlated; however, the amount of correlation gets smaller, the farther the time points are from each other. For example, the first and second time points have a correlation of $\rho$, whereas the first and third time points have a correlation of $\rho^{2}$. Lastly, the first-order moving-average model, $\Sigma=$ $\operatorname{MA}(1)$, depicts that there is a correlation, $\rho=\left(\frac{\theta}{1+\theta^{2}}\right)$, where $\theta$ represents the moving-average coefficient. Note that this is only between adjacent time points (or lag 1). All other correlations are equal to 0 ( $(\mathrm{lag} \geq 2$ ).

At the second level, Eq. 2 provides the regression equations representing potential variation across subjects in the Level-1 coefficients. Equation 2 describes that the baseline performance for subject $j$ from study $k$ equals an overall baseline for study $k, \theta_{00 k}$, plus a random deviation for each subject $j, u_{0 j k}$, in that study. Similarly, Eq. 2 indicates that the overall treatment effect on level, $\theta_{10 k}$; the linear trend in baseline, $\theta_{20 k}$; and the effect of the intervention on the linear trend, $\theta_{30 k}$, all vary over the subjects from study $k: u_{1 j k}, u_{2 j k}$, and $u_{3 j k}$, respectively. At the third level, the variation across studies is modeled using Eq. 3. Equation 3 represents the initial baseline mean outcome score for study $k$ as the overall outcome averaged across all of the studies, 000, plus a random deviation for study $k, v_{00 k}$. And similarly, Eq. 3 represents the average fixed effects across studies: the immediate treatment effect, 100 ; the linear trend in baseline, 200 ; and the effect of the intervention on the linear trend, 300 ; plus the corresponding random deviations for study $k-v_{10 k}, v_{20 k}$, and $v_{30 k}$, respectively. We assume that the Level-2 and Level-3 errors have covariances $\Sigma_{\mathrm{u}}$ and $\Sigma_{\mathrm{v}}$, and that they are independent, identical, and normally distributed.

Initial Monte Carlo studies of this three-level meta-analytic model have shown that estimates of the fixed effects were unbiased, whereas estimates of the variance components were sometimes biased (Moeyaert et al., 2013a). These studies examined a variety of single-case meta-analytic conditions varying in series length, number of participants per study, number of studies meta-analyzed, and the variance decomposition across levels. A limitation of the previous research involving this threelevel meta-analytic model is that the Level-1 error structure, $\Sigma$, was assumed to be $\sigma^{2} \mathrm{I}$ for both data generation and analysis.

$$
\boldsymbol{\Sigma}=\sigma^{2}
$$$$
\boldsymbol{\Sigma}=\operatorname{AR}(1)
$$$$
\boldsymbol{\Sigma}=\mathrm{MA}(1)
$$

$$
\left|\begin{array}{cccc}
\sigma^{2} & & & \\
0 & \sigma^{2} & & \\
0 & 0 & \sigma^{2} & \\
0 & 0 & 0 & \sigma^{2}
\end{array}\right|
$$
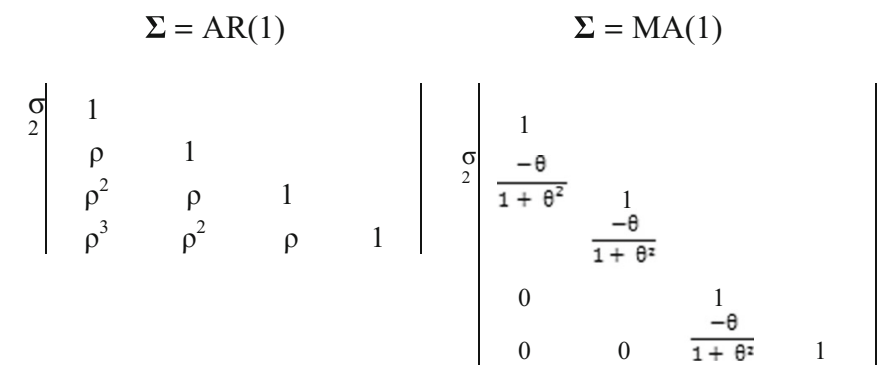

Fig. 1 The three different error specifications for the Level-1 error structure. Furthermore, the figure illustrates the correlations across four time points 


\section{Purpose}

The need to combine study results from single-case designs, which often include small sample sizes, makes this study's purpose unique from that of broader, longitudinal studies. The purpose of this study was to examine the accuracy of parameter estimates in the meta-analysis of single-case data using the previously mentioned three-level model in which the Level-1 error structure is assumed to be AR(1). More specifically, in this study we used Monte Carlo simulation methods to investigate bias in the parameter estimates, both treatment effects and variance components, and to examine the coverage of the corresponding confidence intervals for applications of the model in a variety of conditions, including those in which the assumed AR(1)structure for the Level-1 errors is a correct specification, an overspecification, and a misspecification of the covariance structure.

\section{Method}

Monte Carlo simulation methods were used to examine the parameter estimates for the three-level model when metaanalyzing single-case data. There are many types of singlecase designs, such as the $\mathrm{ABAB}$ design (which is also known as the reversal design) and the multiple-baseline design, which involves time-series data from multiple participants in which an intervention is staggered to occur at different time points within the various series (Ferron et al., 2009). In this study, we simulated multiple-baseline single-case design data under 240 conditions. The conditions varied in series length, the number of participants per study, the number of studies meta-analyzed, the variance among participants, the variance among studies, and the Level-1 error structure [AR(1), MA(1), or independent]. Other than the method used to generate the Level-1 errors, the simulation methods and specific values used were similar to those chosen by Moeyaert et al. (2013a), which were motivated by surveys of single-case design characteristics (Ferron, Farmer, \& Owens, 2010; Shadish $\&$ Sullivan, 2011) and several reanalyses of meta-analyses of single-case studies. Specifically, the series lengths investigated were 10,20, and 40; the number of participants was 4 or 7 ; the number of studies was 10 or 30 ; the variances among participants in the level and slope were 0.5 and 0.05 or 2 and 0.2 , respectively; and the variances across studies in the level and slope were also 0.5 and 0.05 or 2 and 0.2 , respectively. The Level-1 errors were generated with $\sigma^{2}=1$, and were based on one of five combinations of Level-1 residual covariance structure and parameter value scenarios: (1)independence, (2) first-order autoregressive with $\rho=-.3$, (3) firstorder autoregressive with $\rho=.3$, (4) first-order autoregressive with $\rho=.6$, or (5) first-order moving average with $\theta=.3$. The effect of the intervention, or shift in level, coefficient, 100 , was set to 2.0. The effect of the intervention on the linear trend, or shift in slopes, 300, was set to be 0.2 . Fully crossing the values of the two Level-2 residuals' $\left(u_{1 j k}\right.$ and $\left.u_{3 j k}\right)$ variances with the two values for the Level-3 residuals' ( $v_{10 k}$ and $v_{30 k}$ ) variances, along with the five Level-1 error structures, the three series lengths, the two numbers of participants, and the two numbers of studies resulted in a total of $240(=2 \times 2 \times 5 \times 3 \times 2 \times 2)$ conditions for the Monte Carlo study. For each condition, 3, 000 data sets were generated.

In each case, the three-level model was estimated using restricted maximum likelihood via PROC MIXED with the Kenward-Roger degrees-of-freedom method in SAS version 9.3. Ferron, Farmer, and Owens (2010) found that the Kenward-Roger method would be expected to perform the best when there was a complex error structure. Furthermore, the authors summarized their article by suggesting that researchers conducting multiple-baseline studies with multilevel modeling should use the Kenward-Roger method for estimating degrees of freedom (Ferron, Farmer, \& Owens, 2010). The variance components were estimated with a lower bound of 0 , to ensure that the variance was not negative, and the Level-1 error structure was assumed to be first-order autoregressive. Consequently, we were able to examine correct specification of the Level-1 error structure [assuming AR(1)when the structure was $\mathrm{AR}(1)$ ], misspecification [assuming AR(1)when the structure was MA(1)], and overspecification [assuming $\mathrm{AR}(1)$ when the structure was independent].

For each of the 240 conditions, we estimated the relative parameter bias (the difference between the average estimated parameter value and the known parameter value, divided by the known parameter value) for the fixed effect of the treatment on the level (shift in level) and the fixed effect of the treatment on the linear trend (shift in slopes). The relative parameter biases were also examined for the variance components. The confidence interval coverage for the fixed effects was computed as the proportion of the $95 \%$ confidence interval estimates that contained the true parameter value.

\section{Results}

\section{Fixed effects}

Relative parameter bias The relative parameter bias values for the treatment effect on level (shown in Fig. 2) were close to zero. Figure 3 shows the relative biases for the treatment effect on the trend, for which the relative parameter bias values were also close to zero. Hoogland and Boomsma (1998) identified any relative bias greater than 0.05 as being substantial. Only one relative bias estimate exceeded this value. The most extreme outlier in Fig. 3, with a relative bias estimate of 0.0700 , had the following design factors: number of observations $=$ 10 , number of participants $=7$, number of studies $=10$, 


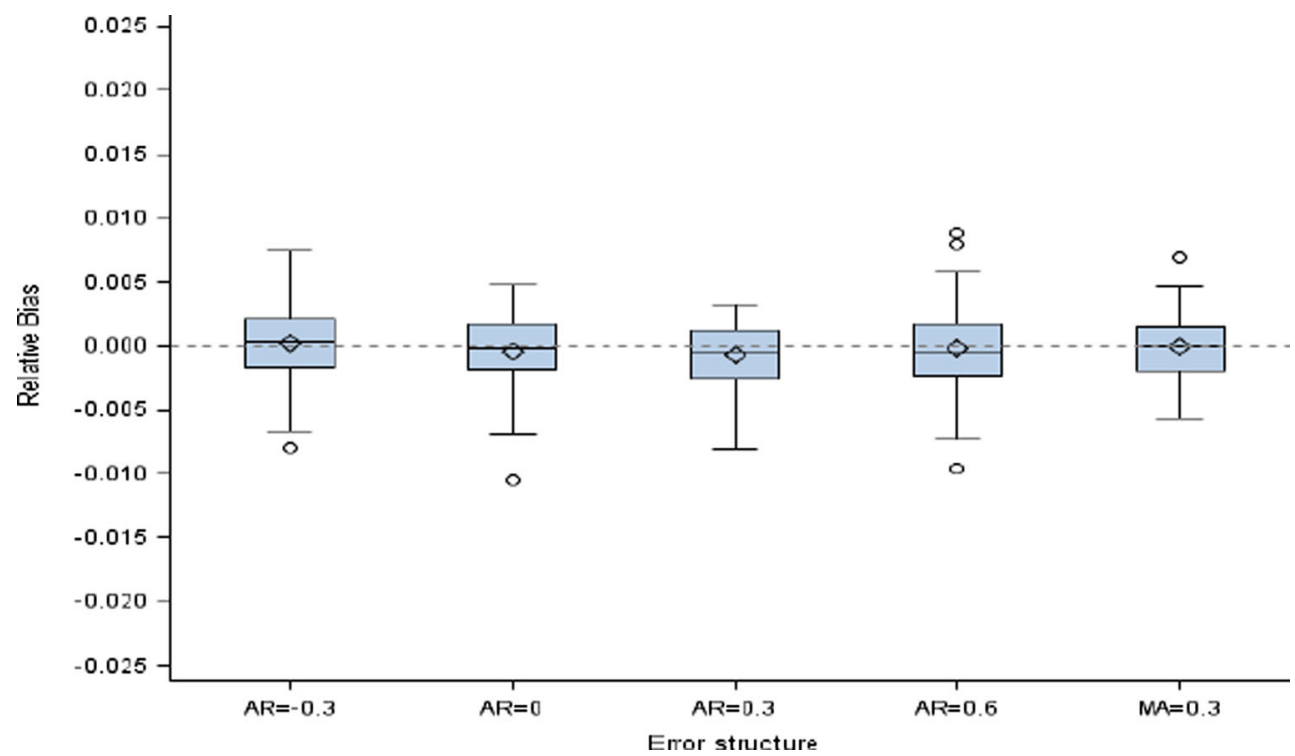

Fig. 2 Boxplots showing the distribution of bias estimates for the shift in level for each of the levels of autocorrelation and the moving-average model

variance in treatment effect (shift in level, among both participants and studies $)=2.0$, variance in treatment on trend (shift in slopes, among both participants and studies) $=0.2$, and autocorrelation parameter $(\rho)=.6$. As with previous studies that had examined the effect of misspecification of the Level-1 error covariance structure in a multilevel model (Ferron et al., 2009; Ferron et al., 2002; Kwok et al., 2007)_although these studies had only looked at the two-level model-the fixedeffect estimates were not appreciably biased.

Confidence interval coverage Figure 4 illustrates boxplots showing the distribution of the coverage rates for the shift-inlevel estimates. The plots show that the average confidence interval coverage rates approached $95 \%$ across all of the levels of autocorrelation, including for the moving-average model, which was the misspecified model. The plots also illustrate that the results fell within Bradley's (1978) robustness criterion for coverage, in that a majority of the conditions fell within his strict criteria, with rates falling between $94.5 \%$ and $95.5 \%$. All of the conditions fell within the liberal criteria, except for two conditions. In one of those conditions the coverage rate was too low, with an estimate of $91.3 \%$. This condition was the same one identified as an outlier in the analysis of relative bias (number of observations $=10$, number of participants $=7$, number of studies $=10$, variance in treatment effect $[$ shift in level, among both participants and studies] $=2.0$, variance in

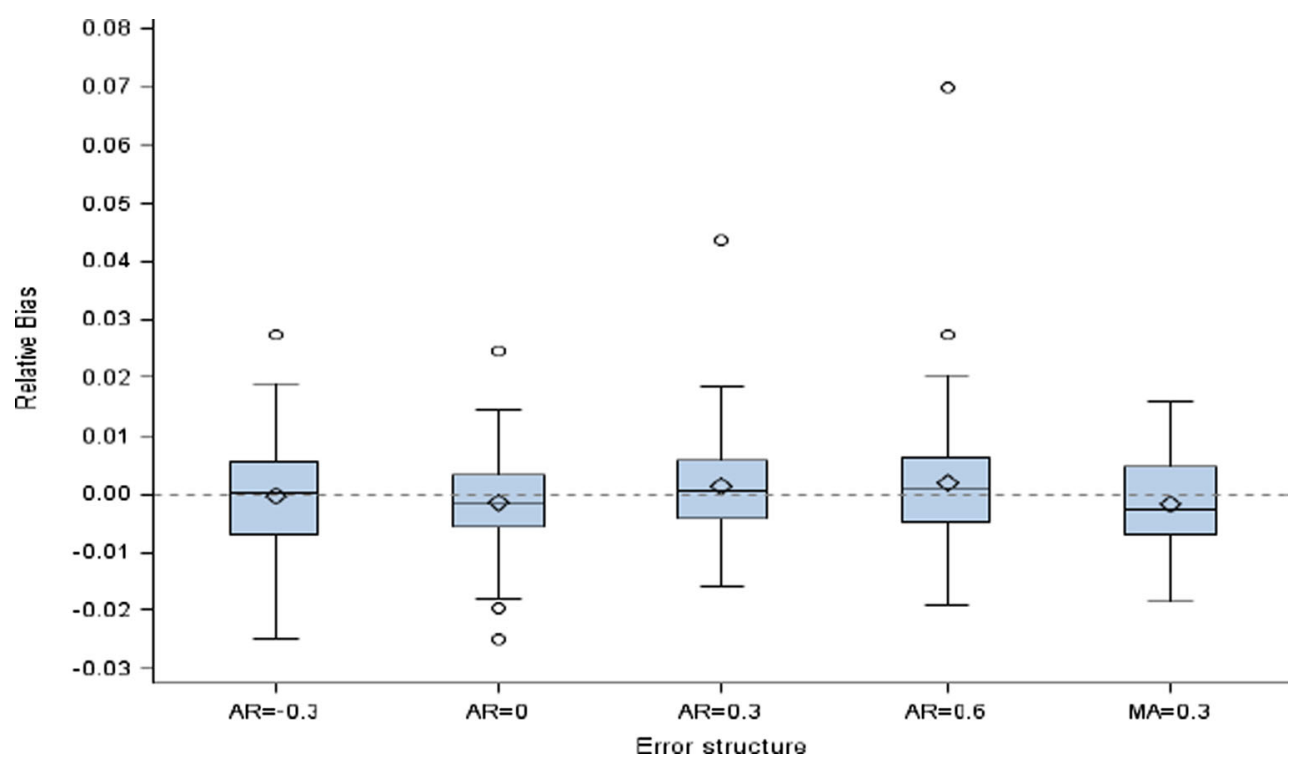

Fig. 3 Boxplots showing the distribution of bias estimates for the treatment effect on trends (shift in slopes) for each of the levels of autocorrelation and the moving-average model 


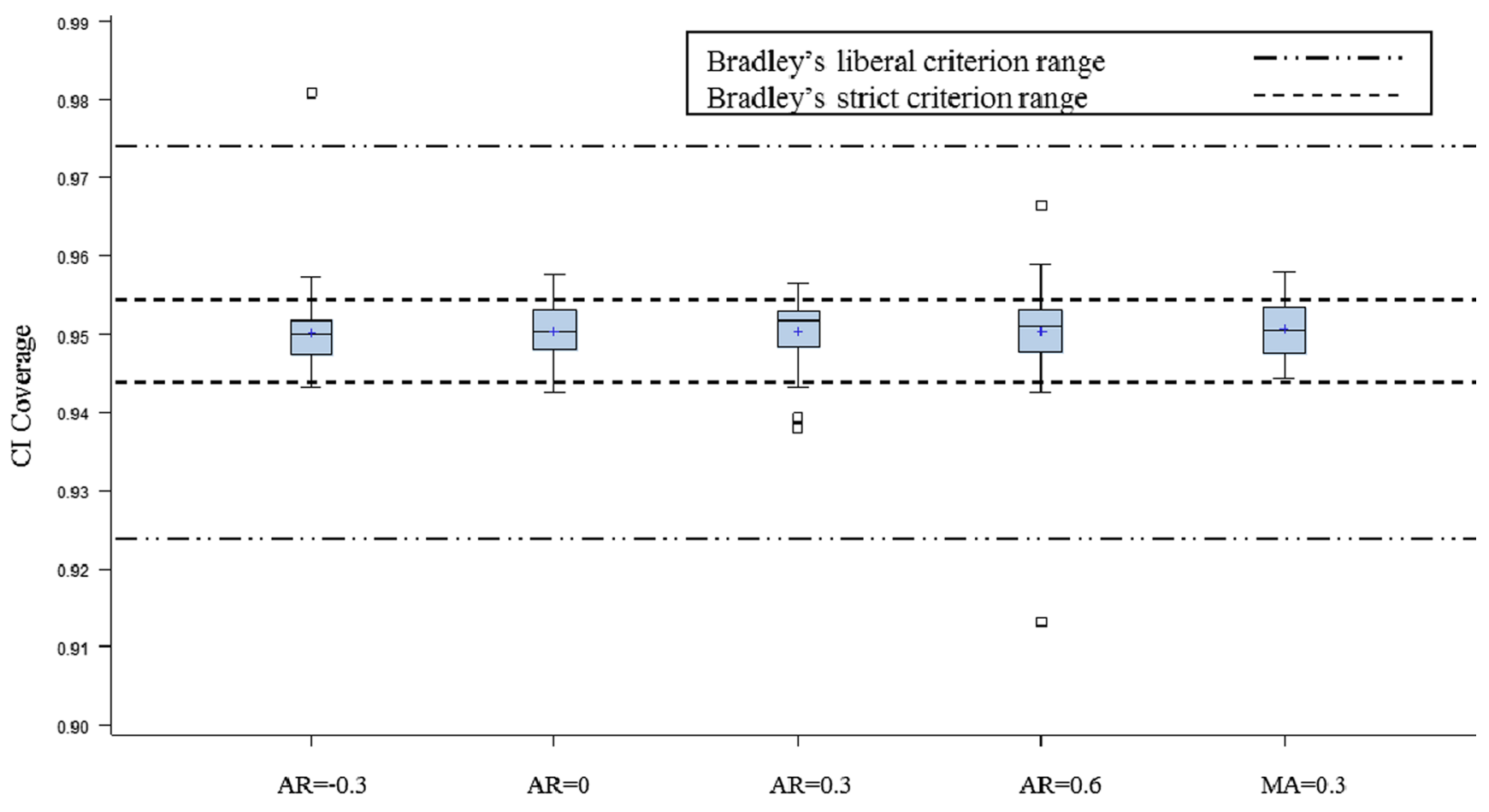

Error structure

Fig. 4 Boxplots illustrating the distribution of confidence interval (CI) coverage estimates for the treatment effect on shifts in level for each of the levels of autocorrelation and the moving-average model

treatment on trend [shift in slopes, among both participants and studies $]=0.2$, and $\rho=.6$ ). In one other condition, too high a coverage rate was found, with a value of $98.1 \%$, with the following design factors: number of observations $=40$, number of participants $=4$, number of studies $=10$, variance in treatment effect (shift in level, among both participants and studies) $=0.5$, variance in treatment on trend (shift in slopes, among both participants and studies) $=0.05$, and $\rho=-.3$.

Figure 5 illustrates boxplots showing the distribution of the coverage estimates for the shift-in-slopes parameter. The plots show that the average confidence interval coverage approached $95 \%$ across all of the generating autocorrelation values, including for the moving-average model, which was the misspecified model. The plot also illustrates that almost all of the conditions fell within Bradley's liberal criterion, with one exception. The condition in which number of observations $=40$, number of participants $=4$, number of studies $=$ 10 , variance in treatment effect (shift in level, among both participants and studies) $=0.5$, variance in treatment on trend (shift in slopes, among both participants and studies) $=0.05$, and $\rho=-.3$ tended to have too high a coverage rate, with an estimate of $98.1 \%$. In sum, the results of the coverage analyses suggest that the model assuming AR(1)performs well for inferences about the fixed effects when it is correctly specified, but that it is also relatively robust when the AR(1)structure represents a misspecification or an overspecification of the Level-1 error structure.

\section{Variance components: Relative parameter bias}

Table 1 contains descriptive statistics describing the distribution of variance component relative parameter bias values for each of the variance components across the three levels of the model. The average relative parameter biases for the Level-2 and Level-3 variance components ranged from 6.80 , for the variance for baseline slopes (Level 2), to 25.72, for the variance component for the baseline shift in level at Level 3. In addition, the range for the relative parameter bias estimates across conditions varied by variance component, with the smallest value for the Level-2 variance for shift in slopes, ranging from -0.23 to $1,620.83$, and the largest value for the Level-3 variance for shifts in level. A further examination of the values revealed that the majority of the variance components seemed reasonable; however, in some instances the variance component estimates were extremely large, such as values similar to $3,777.51$.

Table 1 also contains relative parameter bias estimates for both descriptors of the Level-1 covariance structure. The average relative bias for the autocorrelation parameter was .12 , with values ranging from -.02 to .65 . For the residuals' variances, the average bias was 0.25 , with values ranging from -0.002 to 6.92 . A further investigation of the residual variances revealed two outliers. The more extreme outlier, with a relative parameter bias 


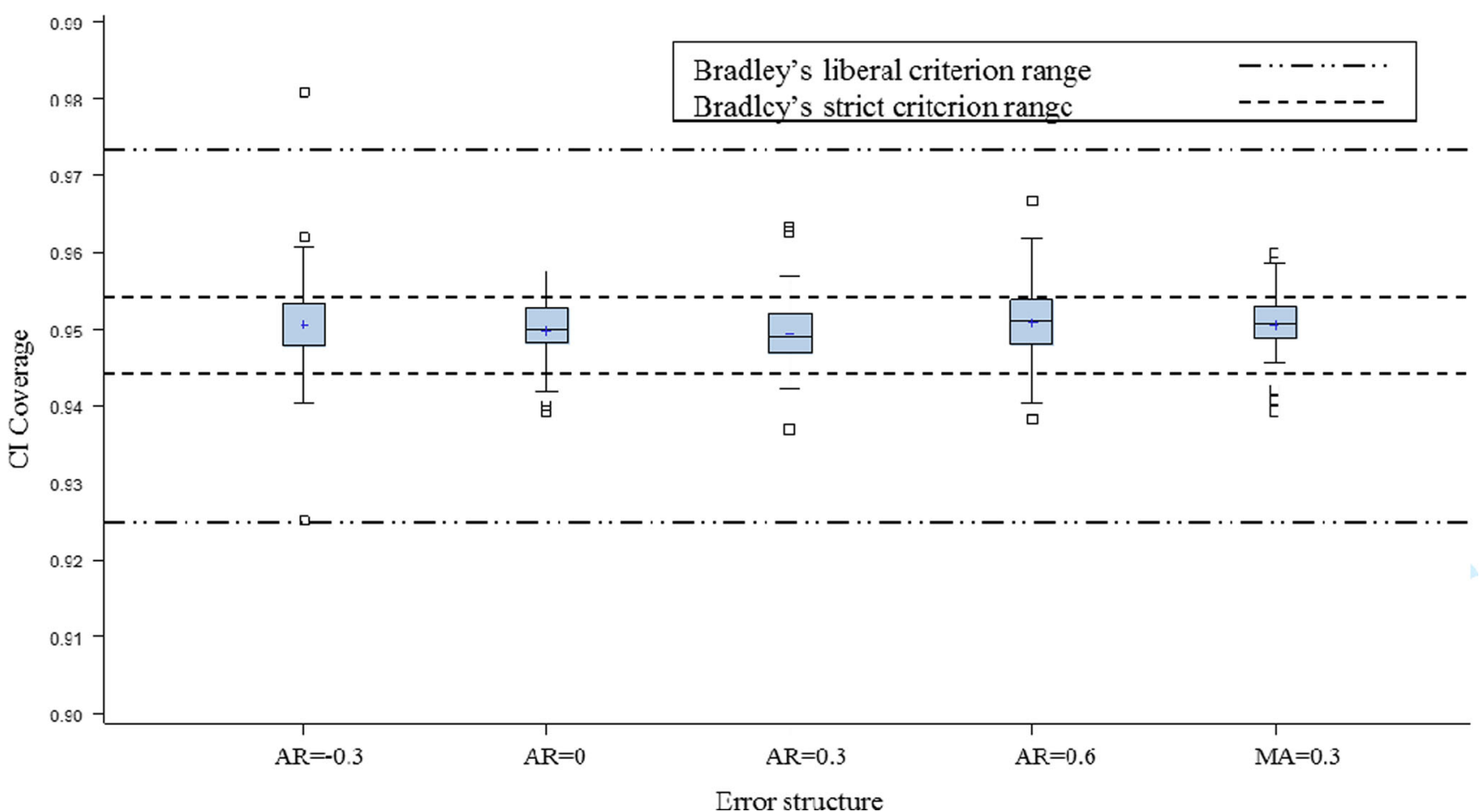

Fig. 5 Boxplots showing the distribution of confidence interval (CI) coverage estimates for the treatment effect on trends for each of the levels of autocorrelation and the moving-average model

estimate of 6.92 (corresponding to $692 \%$ bias), had the following design factors: number of observations $=40$, number of participants $=7$, number of studies $=30$, variance in treatment effect (shift in level, among both participants and studies) $=2.0$, variance in treatment on trend (shift in slopes, among both participants and studies) $=$ 0.2 , and $\rho=-.3$. The other outlier, with a relative bias estimate of 3.42 (or $342 \%$ ), had the following design factors: number of observations $=40$, number of participants $=7$, number of studies $=30$, variance in treatment effect (shift in level, among both participants and studies $)=2.0$, variance in treatment on trend (shift in slopes, among both participants and studies) $=0.2$, and $\rho=0$.

Further analyses were conducted to determine whether or not the extreme bias was systematically associated with any of the study's design factors. Specifically, general linear models were run for all of the upper-level variances. For the Level-2 models, the largest eta-squared value was .0628 for the variance in the baseline slope, for the four-way interaction of Level-1 sample size, Level-2 sample size, Level-3 sample size, and level of autocorrelation. A similar pattern was found for the

Table 1 Statistics describing relative parameter bias values for each of the variance components

\begin{tabular}{|c|c|c|c|c|c|c|c|c|c|c|c|}
\hline & & \multirow[t]{2}{*}{ Mean } & \multirow[t]{2}{*}{ Min } & \multicolumn{5}{|l|}{ Quartiles } & \multirow[t]{2}{*}{ Max } & \multirow[t]{2}{*}{ Skewness } & \multirow[t]{2}{*}{ Kurtosis } \\
\hline & & & & $5 \%$ & $25 \%$ & $50 \%$ & $75 \%$ & $95 \%$ & & & \\
\hline \multirow[t]{4}{*}{ Level Three } & $v_{0 j k}$ & 10.93 & -0.12 & -0.014 & -0.004 & 0.005 & 0.050 & 36.45 & $1,232.47$ & 14.05 & 208.70 \\
\hline & $v_{1 j k}$ & 25.72 & -0.08 & -0.014 & -0.003 & 0.007 & 0.057 & 47.42 & $3,777.51$ & 14.83 & 225.77 \\
\hline & $v_{2 j k}$ & 9.09 & -0.03 & -0.014 & -0.003 & 0.005 & 0.072 & 22.95 & 860.31 & 11.96 & 160.44 \\
\hline & $v_{3 j k}$ & 10.05 & -0.05 & -0.015 & -0.004 & 0.008 & 0.130 & 27.91 & $1,193.71$ & 13.59 & 196.52 \\
\hline \multirow[t]{4}{*}{ Level Two } & $u_{0 j k}$ & 22.57 & -0.19 & -0.10 & -0.016 & -0.003 & 0.007 & 57.61 & $1,308.78$ & 7.86 & 67.48 \\
\hline & $u_{1 j k}$ & 22.16 & -0.12 & -0.07 & -0.010 & -0.001 & 0.014 & 100.59 & $1,091.13$ & 7.46 & 60.77 \\
\hline & $u_{2 j k}$ & 6.80 & -0.07 & -0.02 & -0.010 & 0.000 & 0.020 & 28.55 & 775.76 & 14.11 & 210.20 \\
\hline & $u_{3 j k}$ & 19.16 & -0.23 & -0.11 & -0.010 & 0.000 & 0.060 & 54.28 & $1,620.83$ & 10.50 & 125.98 \\
\hline \multirow[t]{2}{*}{ Level One } & $e_{i j k}$ & 0.25 & -0.002 & 0.0002 & 0.100 & 0.100 & 0.220 & 0.61 & 6.92 & 9.33 & 109.17 \\
\hline & $\rho$ & .12 & -.02 & -.003 & -.0008 & .00005 & .002 & .63 & .65 & 1.51 & 0.30 \\
\hline
\end{tabular}


Level-3 variance component: The largest eta-squared value was .90 , for the design factor that included the fourway interaction of sample size on all three levels crossed with the level of autocorrelation. Therefore, we found no systematic association among the extremely biased variance components and the study's design factors.

\section{Conclusions}

This study's aim was to examine the accuracy of the estimates of the treatment effect parameters in the meta-analysis of single-case data using Van den Noortgate and Onghena's (2008) three-level model. The set of conditions chosen for our study was based on a literature review of single-case designs and the use of multilevel modeling to meta-analyze single-case designs (e.g., Moeyaert et al., 2013a, b; Shadish \& Sullivan, 2011). These conditions, however, represent only some of the possible options. Therefore, the conclusions from this study are limited to contexts in which the conditions mirror those that were examined in this study. In terms of bias and coverage, the fixed-effect estimates of the average treatment effects appeared to be very accurate when the Level- 1 error structure was correctly specified as AR(1). Furthermore, these treatment effect estimates seemed robust to misspecifications of the Level-1 error structure [i.e., specifying AR(1)when the errors were generated to follow a MA(1)structure] as well as robust to overspecification of the Level-1 error structure [i.e., specifying AR(1)when the errors were generated on the basis of an independent model]. Given the small magnitude of bias and the accuracy of the confidence interval coverages, the results are encouraging for applied researchers or meta-analysts seeking to make inferences about the average treatment effects (shift in level and change in slopes) when using the multilevel model to meta-analyze single-case data.

This finding - when coupled with previous work (Ferron et al., 2009; Kwok et al., 2007) that has found that using the simplest Level-1 error structure, the independent model, can lead to faulty inferences when the errors are autocorrelated at the first level of a two-level model-leads us to suggest that researchers should consider using the AR(1)structure when they believe that errors may be autocorrelated. This recommendation is consistent with the recommendations made for more general growth curve modeling applications by Kwok et al. (2007). However, it is also important to further examine the robustness of multilevel models that are applied to singlecase data. Research on general longitudinal models has shown that misspecification of the variance structure at the first level of the model can be partially absorbed in the variance components at a higher level of the model (Ferron et al., 2002; Kwok et al., 2007). It may be, for single-case models, that specification of a more complex Level-2 error structure that allows covariance between the Level-2 errors to be estimated (Moeyaert, 2014; Shadish, Kyse, \& Rindskopf, 2013) can provide greater robustness to not modeling autocorrelation in the Level-1 errors than was reported by Ferron et al. (2009), or it may be that the three-level models that we studied were more robust to nonmodeled autocorrelation in the Level-1 errors because they are based on a more complex variance structure than two-level models.

In related work on the consequences of misspecification of multilevel models for single-case data, Petit-Bois (2014) examined the use of fit indices to select among possible variance structures, and found that although the fit indices often failed to identify the true variance structure, the use of the structure identified by the fit indices led to accurate inferences about fixed effects, whereas Ferron, Moeyaert, Van den Noortgate, and Beretvas (2014) found that - depending on the nature of the error misspecifications caused by history effects - the fixed-effect inferences might or might not be jeopardized. Thus, our finding that the three-level meta-analytic model for single-case data that included estimation of first-level error autocorrelation provided fixed-effect inferences that were robust when the Level-1 error structure was misspecified or overspecified is tempered by the recognition that more research will be needed to fully understand the effects of various types of model misspecification on the fixed-effect inferences made from single-case data.

Additionally, the findings in this study may not apply directly to other single-case designs. One resounding conclusion in previous work has been that more data is better, specifically in terms of the highest level of the model. The model performed better when there were more units at the second level (Ferron et al., 2009), and at the third level when using the meta-analytic model (Petit-Bois, 2014).

For meta-analysts who are primarily interested in the variance components, the results from our study are less promising. The Level-2 and Level-3 residuals' variances tended to show substantial bias in their values, even when the error structure was correctly specified. This result parallels what has been found in other studies of three-level models of single-case data (Moeyaert et al., 2013b; Owens \& Ferron, 2012; Petit-Bois, 2014), and this could possibly be due to the small sample sizes that are used in single-case designs. Future research could examine alternative methods for estimating these models, such as bootstrapping (Ugille, Moeyaert, Beretvas, Ferron, \& Van den Noortgate, 2014) or Bayesian approaches (Shadish, Kyse, \& Rindskopf, 2013), in an effort to reduce the instability in the variance estimates.

In closing, we make some final recommendations: (1)On the basis of the results of this study, we encourage researchers interested in fixed effects (e.g., average change in level, average change in slope) and inferences about those effects (i.e., significance tests, confidence intervals) to consider the explicit modeling of autocorrelation at the first level of a three-level 
model for synthesizing single-case data across cases and studies. (b) On the basis of the results of this study and others (Moeyaert et al., 2013b; Owens \& Ferron, 2012; Petit-Bois, 2014), we encourage caution in interpreting the variance components, particularly the higher-level variance components. (c) On the basis of this study and others (Moeyaert et al., 2013b; Owens \& Ferron, 2012; Petit-Bois, 2014), we encourage researchers to recognize that although fixed effects are estimated without bias even at the smallest sample sizes, as the numbers of observations, cases, and studies being synthesized increase, the confidence interval widths become narrower, leading to more precise estimates of these effects. Therefore, it is recommended that when making methodological decisions, researchers should always attempt to include more data at all levels, but particularly at the third level, in the case of the meta-analytic single-case model.

Author note We gratefully acknowledge support from the Institute of Educational Sciences, U.S. Department of Education, through Grant Number R305D110024. The opinions expressed are those of the authors and do not represent the views of the Institute or the U.S. Department of Education.

\section{References}

Baek, E. K., Moeyaert, M., Petit-Bois, M., Beretvas, S. N., Van den Noortgate, W., \& Ferron, J. M. (2014). The use of multilevel analysis for integrating single-case experimental design results within a study and across studies. Neuropsychological Rehabilitation, 24, 590-606. doi:10.1080/09602011.2013.835740

Beretvas, S. N., \& Chung, H. (2008). A review of meta-analyses of single subject experimental designs: Methodological issues and practice. Evidence-Based Communication Assessment and Intervention, 2, 129-141. doi:10.1080/17489530802446302

Bradley, J. (1978). Robustness? British Journal of Mathematical and Statistical Psychology, 31, 144-152.

Borckardt, J., \& Nash, M. (2014). Simulation modelling analysis for small sets of single-subject data collected over time. Neuropsychological Rehabilitation, 24, 492-506.

Bulté, I., \& Onghena, P. (2008). An R package for single-case randomization tests. Behavior Research Methods, 40, 467-478. doi:10.3758/ BRM.40.2.467

Bulté, I., \& Onghena, P. (2009). Randomization tests for multiple baseline designs: An extension of the SCRT-R package. Behavior Research Methods, 41, 477-485. doi:10.3758/BRM.41.2.477

Edgington, E. S., \& Onghena, P. (2007). Randomization tests (4th ed.). London, UK: Chapman \& Hall.

Ferron, J. M., Bell, B. A., Hess, M. R., Rendina-Gobioff, G., \& Hibbard, S. T. (2009). Making treatment effect inferences from multiplebaseline data: The utility of multilevel modeling approaches. Behavior Research Methods, 41, 372-384. doi:10.3758/BRM.41. 2.372

Ferron, J. M., Dailey, R., \& Yi, Q. (2002). Effects of misspecifying the first-level error structure in two-level models of change. Multivariate Behavioral Research, 37, 379-403.

Ferron, J. M., Farmer, J. L., \& Owens, C. M. (2010). Estimating individual treatment effects from multiple-baseline data: A Monte Carlo study of multilevel-modeling approaches. Behavior Research Methods, 42, 930-943. doi:10.3758/BRM.42.3.930
Ferron, J. M., \& Jones, P. (2006). Tests for the visual analysis of responseguided multiple-baseline data. Journal of Experimental Education, 75, 66-81.

Ferron, J. M., Moeyaert, M., Van den Noortgate, W., \& Beretvas, S. N. (2014). Estimating casual effects from multiple-baseline studies: Implications for design and analysis. Psychological Methods, 19, 493-510. doi:10.1037/a0037038

Ferron, J. M., \& Onghena, P. (1996). The power of randomization tests for single-case phase designs. Journal of Experimental Education, 64, 231-239.

Ferron, J. M., \& Ware, W. (1995). Analyzing single-case data: The power of randomization tests. Journal of Experimental Education, 63, $167-178$.

Greenwood, K., \& Matyas, T. (1990). Problems with the application of interrupted time series analysis for brief single-subject data. Behavioral Assessment, 12, 355-370.

Hedges, L., \& Olkin, I. (1985). Statistical methods for meta-analysis. San Diego, CA: Academic Press.

Hedges, L., Pustejovsky, J., \& Shadish, W. (2012). A standardized mean difference effect size for single case designs. Research Synthesis Methods, 3, 224-239.

Hedges, L., Pustejovsky, J., \& Shadish, W. (2013). A standardized mean difference effect size for multiple baseline designs across individuals. Research Synthesis Methods, 4, 324-341.

Heyvaert, M., \& Onghena, P. (2014). Analysis of single-case data: Randomisation tests for measures of effect size. Neuropsychological Rehabilitation, 24, 507-527.

Hoogland, J., \& Boomsma, A. (1998). Robustness studies in covariance structure modeling: An overview and a meta-analysis. Sociological Methods \& Research, 26, 329-367.

Huitema, B., \& McKean, J. (2000). Design specification issues in timeseries intervention models. Educational and Psychological Measurement, 60, 38-58.

Kazdin, A. (2011). Single-case research designs: Method for clinical and applied settings (2nd ed.). New York: Oxford University Press.

Kratochwill, T., Alden, K., Demuth, D., Dawson, D., Panicucci, C., Arntson, P., \& Levin, J. (1974). A further consideration in the application of an analysis-of-variance model for the intrasubject replication design. Journal of Applied Behavior Analysis, 7, 629-633.

Kratochwill, T., Hitchcock, J., Horner, R., Levin, J., Odom, S., Rindskopf, D., \& Shadish, W. (2010). Single-case designs technical documentation. Retrieved from the What Works Clearinghouse website: http://ies.ed.gov/ncee/wwc/pdf/wwc_scd.pdf

Kwok, O., West, S. G., \& Green, S. B. (2007). The impact of misspecifying the within-subject covariance structure in multiwave longitudinal multilevel models: A Monte Carlo study. Multivariate Behavioral Research, 42, 557-592. doi:10.1080/00273170701540537

Levin, J., Ferron, J., \& Kratochwill, T. (2012). Nonparametric statistical tests for single-case systematic and randomized $\mathrm{ABAB} \ldots \mathrm{AB}$ and alternating treatment intervention designs: New developments, new directions. Journal of School Psychology, 50, 599-624.

Moeyaert, M. (2014). Three-level synthesis of single-subject data: Further developments, empirical validation and applications (Unpublished doctoral dissertation). Leuven: Katholieke Universiteit.

Moeyaert, M., Ferron, J. M., Beretvas, S. N., \& Van den Noortgate, W. (2014). From a single-level analysis to a multilevel analysis of single-case experimental designs. Journal of School Psychology, 52, 191-211. doi:10.1016/j.jsp.2013.11.003

Moeyaert, M., Ugille, M., Ferron, J., Beretvas, S., \& Van den Noortgate, W. (2013a). The three-level synthesis of standardized single-subject experimental data: A Monte Carlo simulation study. Multivariate Behavioral Research, 48, 719-748.

Moeyaert, M., Ugille, M., Ferron, J., Beretvas, S. N., \& Van den Noortgate, W. (2013b). Three-level analysis of single-case experimental data: Empirical validation. Journal of Experimental Education, 82, 1-21. 
Owens, C. M., \& Ferron, J. M. (2012). Synthesizing single-case studies: A Monte Carlo examination of a three-level meta-analytic model. Behavior Research Methods, 44, 795-805. doi:10.3758/s13428011-0180-y

Parker, R. I., Hagan-Burke, S., \& Vannest, K. (2007). Percent of all nonoverlapping data PAND: An alternative to PND. Journal of Special Education, 40, 194-204.

Parker, R. I., Vannest, K. J., \& Davis, J. L. (2011a). Nine non-overlap techniques for single case research. Behavior Modification, 35, 303322.

Parker, R. I., Vannest, K. J., Davis, J. L., \& Sauber, S. B. (2011b). Combining nonoverlap and trend for single-case research: Tau-U. Behavior Therapy, 42, 284-299. doi:10.1016/j.beth.2010.08.006

Petit-Bois, M. (2014). A Monte Carlo study: The consequences of the misspecification of the Level-1 error structure when meta-analyzing single-case designs using a three-level model (Unpublished doctoral dissertation). Tampa, FL: University of South Florida.

Scruggs, T. E., Mastropieri, M. A., \& Castro, G. (1987). The quantitative synthesis of single-subject research: Methodology and validation. Remedial and Special Education, 8, 24-33.

Shadish, W. R., Kyse, E. N., \& Rindskopf, D. M. (2013). Analyzing data from single-case designs using multilevel models: New applications and some agenda items for future research. Psychological Methods, 18, 385-405. doi:10.1037/a0032964
Shadish, W., \& Sullivan, K. (2011). Characteristics of single-case designs used to assess intervention effects in 2008. Behavior Research Methods, 43, 971-980. doi:10.3758/s13428-0110111-y

Swaminathan, H., Rogers, H., \& Horner, R. (2014). An effect size measure and Bayesian analysis of single-case designs. Journal of School Psychology, 52, 213-230.

Toothaker, L., Banz, M., Noble, C., Camp, J., \& Davis, D. (1983). N=1 designs: The failure of ANOVA-based tests. Journal of Educational Statistics, 4, 289-309.

Ugille, M., Moeyaert, M., Beretvas, S. N., Ferron, J., \& Van den Noortgate, W. (2014). Bias corrections for standardized effect size estimates used with single-subject experimental designs. Journal of Experimental Education, 82, 358-374.

Van den Noortgate, W., \& Onghena, P. (2003). Combining single-case experimental data using hierarchical linear models. School Psychology Quarterly, 18, 325-346.

Van den Noortgate, W., \& Onghena, P. (2008). A multilevel metaanalysis of single subject experimental design studies. Evidence-Based Communication Assessment and Intervention, 2, 142-151.

Wolfinger, R. (1993). Covariance structure selection in general mixed models. Communications in Statistics, Simulation and Computation, 22, 1079-1106. 